N. E. Piskunov, W. W. Weiss, D. F. Gray, eds.

\title{
Maser Observations of Cool Stars
}

\author{
E.M.L. Humphreys \\ Onsala Space Observatory, Chalmers University of Technology, Sweden, \\ S-43992
}

\begin{abstract}
.
$\mathrm{SiO}, \mathrm{H}_{2} \mathrm{O}$ and $\mathrm{OH}$ masers are ubiquitous in the circumstellar envelopes (CSEs) of oxygen-rich, red giant stars. Radio interferometry allows the imaging of stellar maser emission down to sub-milliarcsecond scales. Such observations reveal asymmetry, inhomogeneity and apparent clumpiness in the extended atmosphere and surrounding envelope of the star. The studies place constraints on processes which are seldom included in models. Here, I review the observational data on stellar masers and discuss their implications for our understanding of the mass-loss and evolution of red giant stars.
\end{abstract}

\section{Introduction}

$\mathrm{SiO}, \mathrm{H}_{2} \mathrm{O}$ and $\mathrm{OH}$ masers provide an excellent method of studying the CSEs of M-type supergiants and of red giants on the Asymptotic Giant Branch (AGB). Firstly, each of the maser lines of these molecules requires different excitation conditions for producing bright maser emission, hence they probe different regions of the extended CSE built up by long-period variables such as Miras, Semi-Regulars and OH/IR stars, see Figure 1. Secondly, since masers are compact, of high brightness temperature, their emission is ideal for study using radio interferometers which can provide angular resolutions hundreds of times greater than that of the HST. For example, $\mathrm{SiO}$ masers at $43 \mathrm{GHz}$ can now be imaged with an angular resolution of $250 \mu$ as and with a velocity resolution of $0.1 \mathrm{kms}^{-1}$ using the Very Long Baseline Array (VLBA). Finally, maser emission varies during the stellar pulsation cycles of AGB stars. Thus time series observations of stellar masers provide an unprecedented view of the gas dynamics and physical conditions surrounding such mass-losing stars as a function of stellar phase. Below I outline the observational data on stellar masers, starting from emission originating nearest to the photosphere and working outwards.

\section{SiO Masers}

$\mathrm{SiO}$ maser emission has been detected in a range of rotational transitions, from $J=1-0(43 \mathrm{GHz})$ up to $J=10-9(430 \mathrm{GHz})$, within vibrational states $v=0$ to $v=4$ (e.g. Pardo et al. 1998; Bieging et al. 2000). For clarity, Figure 2 shows an energy level diagram for $\mathrm{SiO}$. 


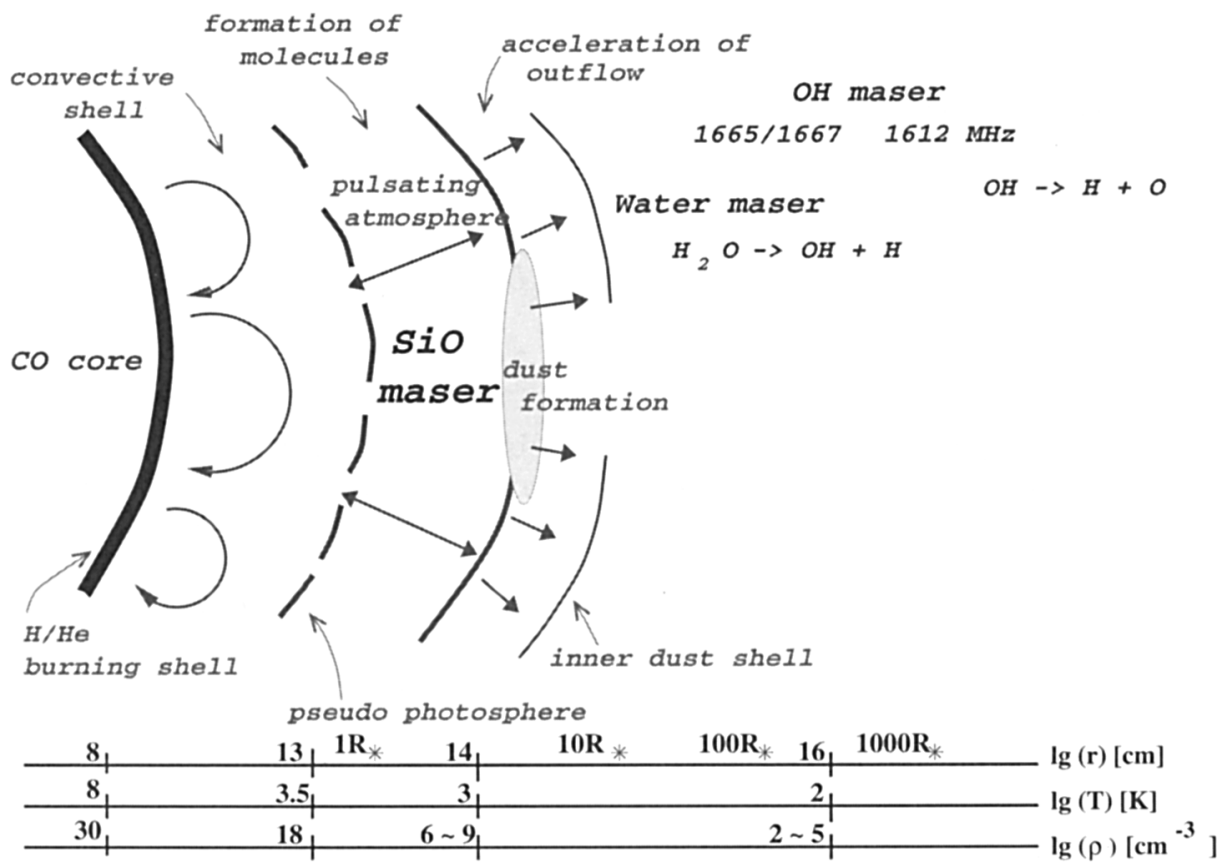

Figure 1. Schematic of an AGB circumstellar envelope. The carbon and oxygen degenerate core is surrounded by layers of $\mathrm{H}$ and $\mathrm{He}$, followed by an extended atmosphere region, from which $\mathrm{SiO}$ maser emission is believed to originate. Water and hydroxyl masers probe regions further from the central star respectively. After Le Bertre (1997).

\subsection{Interferometry Studies}

At the resolution of the VLBA, $\mathrm{SiO}$ maser emission is distributed in a ringlike structure of emitting spots, within a few stellar radii (several AU) of the assumed stellar position. Diamond et al. (1994) were the first to resolve such emission structure for $v=1 J=1-0$ emission towards TX Cam and U Her and show that stellar $\mathrm{SiO}$ masers amplify tangentially, see Figure 3. Tangential amplification (i.e. tangential to the radius vector of the star) is the norm for $\mathrm{SiO}$ masers since high radial velocity gradients in the extended atmosphere region (see Woitke and Höfner, this proc.) severely limit the path length over which the maser is able to continue amplification, usually referred to as the 'velocity coherence' length. Earlier, European Very Long Baseline Interferometry (VLBI) observations had already placed limits on the size of stellar $\mathrm{SiO}$ maser emission spots at $\sim 10^{12} \mathrm{~cm}$ (Colomer et al. 1992). However, it should be noted that some tens of percent of the single-dish maser flux are missing in these high spatial resolution observations. More extended emission structures, resolved out on VLBA baselines, must also be present and require investigation.

Since then, maser rings have been found to be a common phenomenon, via imaging at 43 and $86-\mathrm{GHz}$ towards several stars - (for example Greenhill et al. 


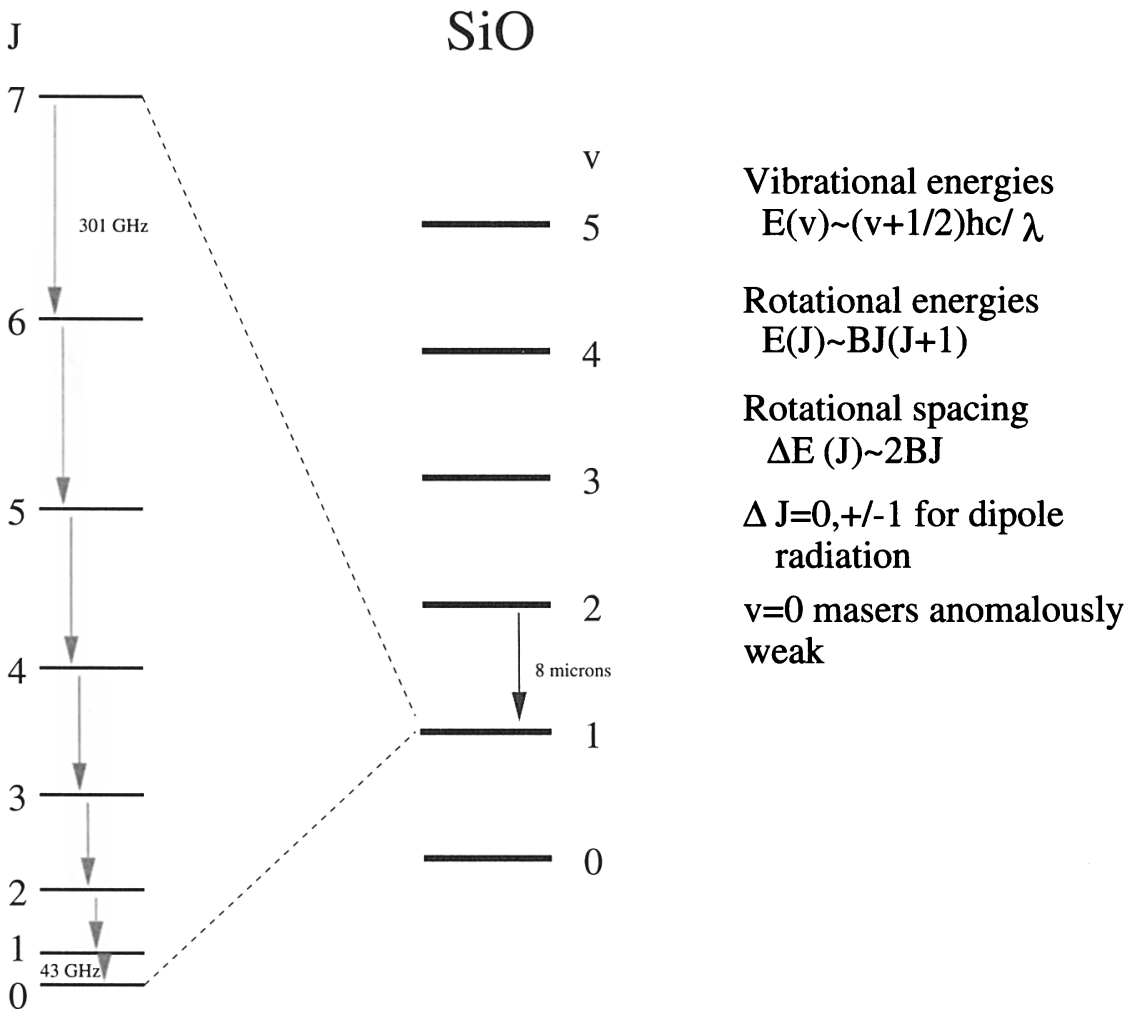

Figure 2. Energy level diagram for SiO.

1995 for $v=1 J=1-0$ masers in VX Sgr; Boboltz et al. 1997 for $v=1$ $J=1-0$ masers in R Aqr; Doeleman et al. 1998 for $v=1 J=2-1$ masers in VX Sgr; Desmurs et al. 2000 for $v=1 \& 2 J=1-0$ masers in TX Cam \& IRC+10011; Yi et al. 2002 for $v=1 \& 2 J=1-0$ masers in TX Cam \& R Cas). Such observations firmly place the $\mathrm{SiO}$ masers in the extended atmosphere between the stellar photosphere and the inner radius of the circumstellar dust shell (Greenhill et al. 1995). Thus energy and chemical requirements for SiO are fulfilled: close to the star in warm, dense regions for populating energy levels $>1800 \mathrm{~K}$ above ground state, and placing masers in regions of high gas phase $\mathrm{SiO}$ abundance, within the radius at which dust condensation takes place. $\mathrm{SiO}$ masers are believed to lie just beyond a stellar "radio photosphere", at around $2 \mathrm{R}_{*}$ (Reid \& Menten 1997).

All these data indicate the inhomogeneity of the extended atmosphere, the cause of which is unknown. A proposed mechanism for the formation of maser clumps has been via thermal instabilities resulting from infrared band 'runaway' cooling by $\mathrm{SiO}$ and $\mathrm{CO}$ (Cuntz \& Muchmore 1994), but this appears to have been discounted (see Woitke, this proc.). Another route to clump formation could be the magnetohydrodynamical Parker instability (see Hartquist \& Dyson 1997), however this would require a magnetic field of around $50 \mathrm{G}$. The extended atmosphere region is also likely to be strongly turbulent. The significance for 

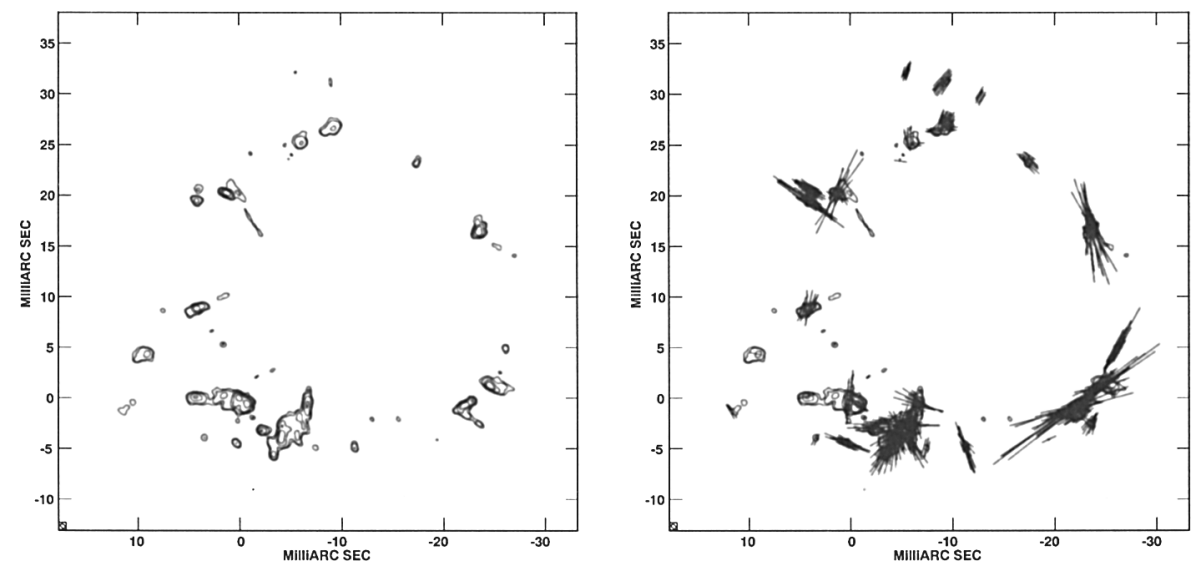

Figure 3. $\quad \mathrm{SiO}$ maser emission in TX Cam $(v=1, J=1-0$ emission at $43 \mathrm{GHz}$ obtained using the VLBA): on the left: total intensity contours (Stokes I); on the right: the linear polarization map. After Kemball \& Diamond (1997).

masers is that only a limited number of lines of sight are available in a turbulent medium for masers to propagate for substantial lengths and therefore to build up high intensities. This is likely to be an important contributory feature in causing intense masers to form an incomplete ring of structures about the central star.

\subsection{Time Variations in the Structure of SiO Maser Emission}

The maser ring is not static, its diameter changes with stellar phase. The first proper motion measurements showed the maser ring in the symbiotic Mira $\mathrm{R}$ Aqr in contraction as the intensity of the ring faded, with an average velocity of $\sim 4 \mathrm{kms}^{-1}$. These observations covered around a quarter of the stellar cycle

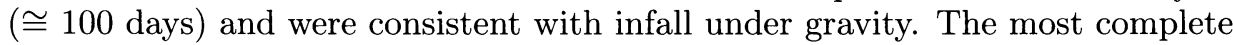
multi-epoch data is the monitoring of the $v=1 J=1-0$ masers in TX Cam over $>1.5$ cycles by Diamond \& Kemball. Both infalling and outflowing gas is observed, but expansion dominates the dynamics. For example, over a nine month period in 1998, the ring had expanded by $>10 \%$ in some directions (Diamond $\&$ Kemball 1999, 2001). Some of the maser components move outwards with a constant velocity of $\sim 10 \mathrm{kms}^{-1}$. These observations provide strong evidence for shocks. Disruption to the maser ring appears to occur at minimum maser light (Diamond, private communication). Around this time a new maser ring is observed to form inside the radius of the previous ring.

\subsection{Multi-Transition Observations}

Ring radii also appear to vary with transition. Towards a few red giants, there is a clear average spatial separation between the $v=1 J=1-0$ and the $v=2 J=1-0$ maser rings, when observed at high enough spatial resolution ( $\sim 0.5$ mas). The separation is $<0.5 \mathrm{AU}$, the $v=2$ masers lying inside the corresponding $v=1$ features (Desmurs et al. 2000; Yi et al. 2002). These 
data are very interesting for constraining the simulations described in Section 5. These simulations predict that the amount of separation of the rings is a phase-dependent quantity.

\subsection{Polarization Structure}

In the VLBA data there is a highly ordered polarization pattern, see Figure 3. Most $\mathrm{SiO}$ maser spots are linearly polarized in a direction which is tangential to the maser ring (Kemball \& Diamond 1997, Diamond \& Kemball 2001; Desmurs et al. 2000). The degree of linear and circular polarization varies substantially between components. When averaged over the shell, linear polarization is at around the $30 \%$ level and circular polarization is $\sim 3 \%$. However, Diamond $\&$ Kemball (2001) show clearly that the south east portion of the ring can also display a smaller. additional circular linear polarization structure, the source of which is unknown.

Ambiguity in the interpretation of $\mathrm{SiO}$ maser polarization arises as $\mathrm{SiO}$ is non-paramagnetic. Zeeman splitting would be smaller than the SiO thermal linewidth, for gas kinetic temperatures say around $1500 \mathrm{~K}$, unless the magnetic field is of the order $\sim 10^{3} \mathrm{G}$. Taking a Zeeman interpretation, the linear polarization pattern provides an indication of the magnetic field structure and the observed circular polarization implies a magnetic field strength of 5 - $10 \mathrm{G}$ (Kemball \& Diamond 1997; Elitzur 1996). For a field of this strength, the magnetic pressure will greatly exceed the gas thermal pressure for the conditions expected in the $\mathrm{SiO}$ maser zone (see Barvainis et al. 1987). In this case the magnetic field would be the dominant force in determining the kinematics of maser cells. Blackman et al. (2001) describe how strong magnetic fields can be produced via a dynamo at the interface between a rapidly rotating core and a more slowly rotating stellar envelope in AGB stars.

Under the non-Zeeman interpretation, the observed linear polarization is due to anisotropy in the radiative pumping of $\mathrm{SiO}$ masers by stellar photons (Western \& Watson 1983). In this scenario the quantization axis is provided by the pumping process itself, the radial direction of the stellar IR pump photons, rather than by a magnetic field. Tangential polarization then arises as molecules rotating in the tangential plane preferentially absorb the stellar photons. This mechanism is clearly described by Desmurs et al. (2000). Given the observed linear polarization, Wiebe \& Watson (1998) show that the circular polarization can be produced if the direction of a magnetic field of only $>30 \mathrm{mG}$ changes in orientation along the line of sight.

\subsection{Rotation in the $\mathrm{SiO}$ Maser Zone?}

Interpretations for rotation in the $\mathrm{SiO}$ maser zone have been given from observations of a few stars - NML Cyg (Boboltz \& Marvel 2000); IK Tau (Boboltz \& Diamond 2000); R Aqr (Hollis et al. 2000) and VX Sgr (Doeleman et al. 1998). Towards the supergiant NML Cyg, the $v=1 J=1-0$ spectrum shows an unusual double-peaked profile, which in conjunction with the spatial velocity distribution, could be interpreted as rotation of the maser shell with a velocity of $\mathrm{V}_{m} \sin i \approx 11 \mathrm{kms}^{-1}$. 

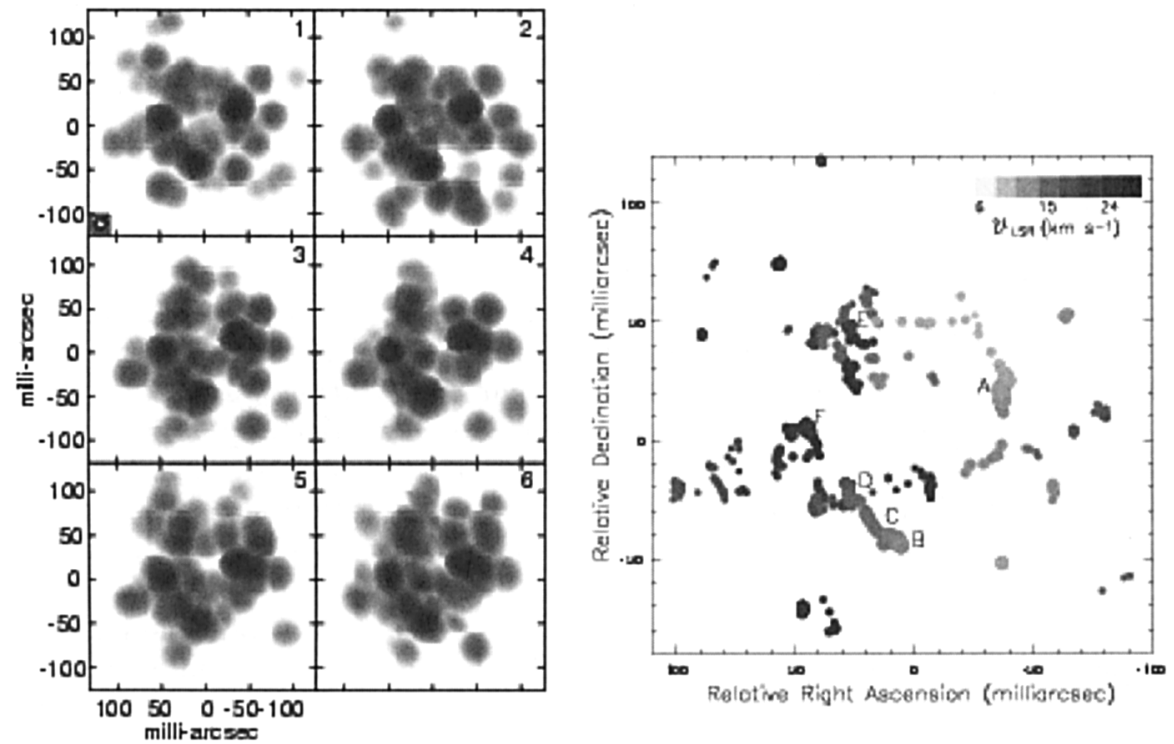

Figure 4. $22 \mathrm{GHz}$ water maser emission towards RT Vir: on the left: Maps of total $22 \mathrm{GHz}$ flux from RT Vir for 6 epochs; on the right: individual maser components at epoch 1 . The shade shows velocity and the symbol diameter is proportional to $\log$ (flux density). After Richards et al. (1999).

\section{3. $\mathrm{H}_{2} \mathrm{O}$ Masers}

The most well-studied $\mathrm{H}_{2} \mathrm{O}$ maser line is that at $22 \mathrm{GHz}$ arising from the $616 \rightarrow$ $5_{23}$ transition in the ground vibrational state of ortho- $\mathrm{H}_{2} \mathrm{O}$. The upper level of this transition lies at an $\mathrm{E}_{u} / \mathrm{k}$ of $\sim 650 \mathrm{~K}$. MERLIN (milli-arcsecond resolution; velocity resolution $0.1 \mathrm{kms}^{-1}$ ) and VLA interferometry data have provided the evidence that the stellar $\mathrm{H}_{2} \mathrm{O}$ maser emission at $22 \mathrm{GHz}$ is located in a shell expanding from AGB stars (e.g. Reid \& Menten 1990; Bowers \& Johnston 1994; Yates et al. 1994; Colomer et al. 2000). The shell appears clumpy and incomplete at these resolutions. Emission originates from the inner parts of the CSE of Mira-type stars, from regions out to $\sim 10^{15} \mathrm{~cm}$, which are comparable in extent to those in which dust grains form and grow, and in which the expanding envelope has not yet reached terminal velocity. $22 \mathrm{GHz}$ masers are believed to probe stellar gas in which acceleration away from the star takes place via radiation pressure on dust and subsequent gas-grain collisions (Chapman \& Cohen 1986). Other $\mathrm{H}_{2} \mathrm{O}$ masers are commonly found in the evolved circumstellar environment. For example, maser emission at 321,325 and $183 \mathrm{GHz}$ (Menten et al. 1990; Menten \& Melnick 1991; Yates et al. 1995; González-Alfonso et al. 1998).

$22 \mathrm{GHz}$ masers result from collisional excitation followed by radiative decay, thus maser components are expected to lie in a shell whose inner and outer 
boundaries are determined by collision rates. At the inner boundary, collision rates quench the maser inversion by thermalising the energy level populations of molecules in the gas. Whilst at the outer boundary collision rates are too low to pump the $22 \mathrm{GHz}$ inversion. In addition, photolysis by the interstellar radiation field acts to reduce $\mathrm{H}_{2} \mathrm{O}$ abundance in the outer envelope.

Figure 4 shows MERLIN observations of the $22 \mathrm{GHz}$ masers of the SemiRegular variable RT Vir. The data were taken over six epochs over a $\sim 3$ month period by Richards et al. (1999). The unfilled ring structure indicates that the brightest maser emission is tangentially amplifying in this star (for stars of higher mass loss rate, radial amplification occurs). The average angular size of a maser component/feature is $2.3 \pm 1.0 \times 10^{13} \mathrm{~cm}$. Richards et al. (1999) find that the components reside in a thick shell whose inner and outer radii and expansion velocities are given by: $\mathrm{r}_{i}=6.9 \times 10^{13} \mathrm{~cm}, \mathrm{r}_{o}=28.8 \times 10^{13} \mathrm{~cm} ; \mathrm{v}_{i}=$ $3.5 \mathrm{kms}^{-1}, \mathrm{v}_{o}=11.0 \mathrm{kms}^{-1}$. However, they find that the outflow deviates from spherical symmetry and it is possible that the star loses mass asymmetrically. They also conclude that $\mathrm{H}_{2} \mathrm{O}$ masing clouds are about 50 times denser than their surroundings, which is consistent with the data in Richards et al. (1996, 1998).

\section{1. $\mathrm{H}_{2} \mathrm{O}$ Polarization}

As for $\mathrm{SiO}$, the Zeeman splitting of $\mathrm{H}_{2} \mathrm{O}$ would be extremely small for the maximum field strengths likely in that region of the CSE (a few hundred $\mathrm{mG}$ ), only $\approx 10^{-3}$ times the typical Gaussian line width of the $\mathrm{H}_{2} \mathrm{O}$ maser line. However, it is possible to detect the splitting using high spectral resolution polarization observations and Vlemmings et al. (2001,2002) have made such measurements for several stars. The values they obtain are $\approx 150 \mathrm{mG}-500 \mathrm{mG}$ for the 3 supergiants measured and for the Mira in their sample, U Her, $\approx 1.5 \mathrm{G}$. However, an non-Zeeman interpretation for this circular polarization is also given in Nedoluha \& Watson (1990).

\section{OH Masers}

$\mathrm{OH}$ masers reside in the outer CSE, in the fully-developed stellar wind. In red giant winds, the $\mathrm{OH}$ maser mainlines at 1665 and $1667 \mathrm{MHz}$ exist in the region $\sim 10^{15}-10^{16} \mathrm{~cm}$, whereas the $1612 \mathrm{MHz}$ masers occur in larger shells at $\sim 10^{16} \mathrm{~cm}$. Figure 5 shows the double-peaked intensity profiles indicative of the radial 'front-back' amplification process for $\mathrm{OH} 1612 \mathrm{MHz}$ masers, the velocity separation between the peaks centering on the stellar velocity. Figure 5 also shows the MERLIN interferometry data for the velocity ranges $a, b, c$ and $d$ marked on the intensity profile. The incomplete and clumpy appearance of the $\mathrm{OH}$ maser shell indicates the deviations from pure spherical symmetry present also in this region of the CSE.

\subsection{OH Polarization}

Zeeman splitting of the $\mathrm{OH}$ lines shows the magnetic field strength in this relatively outer region of the CSE is $\approx 1 \mathrm{mG}$ for Miras (e.g. Szymczak \& Cohen 1997). There is little dispute of the Zeeman origin of the polarization for this 

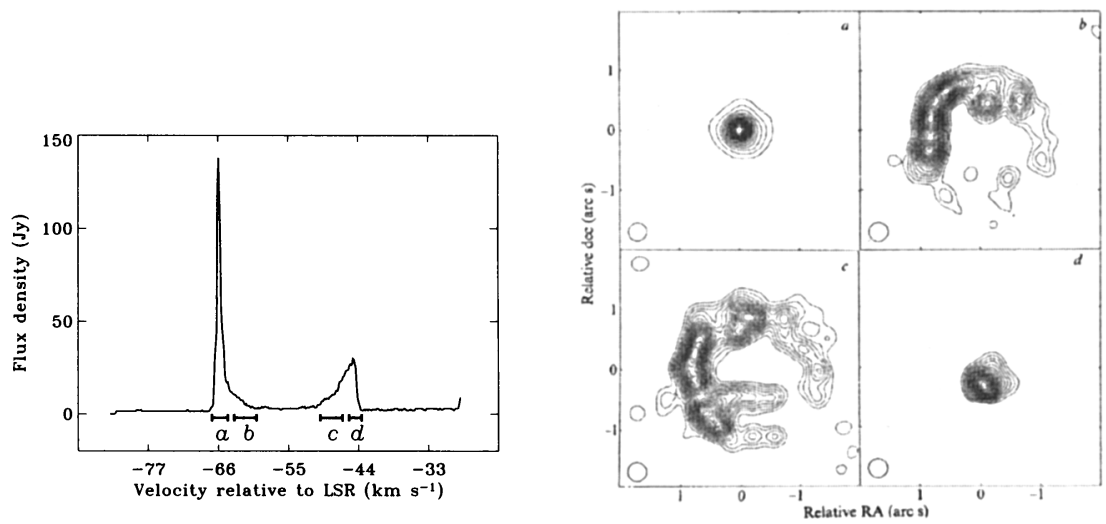

Figure 5. $\quad \mathrm{OH} 1612 \mathrm{MHz}$ maser profile and channel maps of the star OH127.8 (after Booth et al. 1981)

maser species. On the basis of the polarization measurements for all the three maser species, Vlemmings et al. (2002) favour a solar-type magnetic field structure for the CSEs of AGB stars.

\section{Maser Simulations using dynamical stellar models}

In order to investigate the effect of AGB stellar pulsation on stellar masers, Humphreys et al. $(1996,2001,2002)$ couple $\mathrm{SiO}$ and $\mathrm{H}_{2} \mathrm{O}$ maser models to a MMira hydrodynamic pulsation model (Bowen 1988). In calculations performed around every two weeks during the cycle of the model star, VLBI and singledish data can be well-reproduced qualitatively. $\mathrm{SiO}$ masers form in a tangentially amplifying ring within a few stellar radii. $\mathrm{SiO}$ single-dish lineshapes, of extent around $10 \mathrm{~km}^{-1}$, form a single dominant peak near the stellar velocity and maser emission from $v=1-v=3$ up to $J=10-9$ is found. Low-intensity linewings, which exceed the outflow velocity, also occur as a phase-dependent phenomenon, from shocked gas in the extended atmosphere in a model which does not include rotation. Gray \& Humphreys (2000) predict that the spatial separation between the $v=1 \& 2 J=1-0$ maser rings is a stellar phase-dependent quantity, varying between $0.1-0.3 \mathrm{AU}$ during the stellar cycle for a Mira-type star.

The pulsation model has some quantitative drawbacks e.g. the contrast between minimum and maximum maser intensity is overpredicted in these simulations. Some of these problems can largely be attributed to the use of a spherically symmetric model which does not include time-dependent chemistry. Many of the $\mathrm{H}_{2} \mathrm{O}$ maser features are also reproduced by the simulations.

\section{Outlook}

Multi-epoch and polarization imaging of the masers in AGB stars provides invaluable data on the physical conditions in CSEs. We now have better prospects 
than ever for understanding the complex processes governing stellar wind formation and the mass loss process in such stars.

The challenge is to interpret the observations in terms of the temperature, number density, dynamics and magnetic field structure of the stellar extended atmosphere and CSE. Maser observations may indicate the need to incorporate the effects of stellar magnetic fields, clump formation, non-spherical symmetry and perhaps rotation in AGB dynamical models. However, further analyses are required in order to understand fully the cause of polarization for the $\mathrm{SiO}$ and $\mathrm{H}_{2} \mathrm{O}$ maser emission, and other means of verifying the potentially significant $\mathrm{B}$ fields involved should also be sought.

In connection to the status of circumstellar maser modelling and simulations, it is encouraging that existing simulations are now able to reproduce many observed characteristics of circumstellar maser emission. New generation maser codes, which calculate radiative transfer accurately (e.g. using Accelerated Lambda Iteration) for many level systems, are under development and hydrodynamical models which incorporate time-dependent dust formation and chemistry are being produced for O-rich stars. These devlopments will yield increasingly accurate model predictions for stellar masers.

Acknowledgments. I am very grateful to the Swedish Foundation for International Cooperation in Research and Higher Education (STINT) for funding my position and to the LOC for providing travel funds to the meeting. I thank to Jiyune Yi and Malcolm Gray for providing Figures 1 \& 2 respectively, and all the authors whose results are shown in this paper.

\section{References}

Barvainis, R., McIntosh, G., Predmore, C. R. 1987, Nature, 329, 613

Bieging, J.H., Shaked, S., Gensheimer, P. D. 2000, ApJ, 543, 897

Blackman, E.G., Frank, A., Markiel, J.A., et al., 2001, Nature, 409, 585

Boboltz, D., Diamond, P.J., Kemball, A.J. 1997, ApJ, 487, L147

Boboltz, D.A., Diamond P.J. 2000, 197, 4507

Boboltz, D.A., Marvel, K.B. 2000, ApJ, 545, L149

Booth, R., Norris, R.P., Porter, N.D., Kus, A.J. 1981, Nature, 290, 382

Bowen, G. 1988, ApJ, 329, 299

Bowers, P. F., Johnston, K. 1994, ApJS 92, 189

Cernicharo, J., Bujarrabal, V., Santaren, J. L. 1993, ApJ, 407, L33

Chapman, J.M., Cohen, R.J. 1986, MNRAS 220, 513

Colomer, F., Graham, D. A., Krichbaum, T. P., et al. 1992, A\&A, 254, L17

Colomer, F., Reid, M.J., Menten, K.M., Bujarrabal, V. 2000, A\&A 355, 979

Cuntz, M., Muchmore, D.O. 1994, ApJ, 433, 303

Desmurs, J.F., Bujarrabal, V., Colomer, F., Alcolea, J. 2000, A\&A, 360, 189

Diamond, P. J. \& Kemball, A. J. 1999, in IAU 191, 195

Diamond, P. J. \& Kemball, A. J. 2001, in IAU 205, 252

Diamond, P.J., Kemball, A.J, Junor, W., et al. 1994, ApJ, 430, L61 
Doeleman, S.S., Lonsdale, C.J., Greenhill, L.J. 1998, ApJ, 494, 400

Elitzur, M. 1996, ApJ, 457, 415

González-Alfonso, E., Cernicharo, J., Alcolea, J., Orlandi, M.A. 1998, A\&A 334, 1016

Gray, M.D. \& Humphreys, E.M.L. 2000, New Ast., 5, 155

Greenhill, L.J., Colomer, F., Moran, J.M., et al. 1995, ApJ, 449, 365

MNRAS, 243, 480

Hartquist, T.W., Dyson, J.E. 1997, A\&A, 319, 589

Hollis, J. M., Pedelty, J. A., Forster, J. R., et al. 2000, ApJ, 543, L81

Humphreys, E.M.L., Gray, M.D., Yates, J.A., et al. 1996, MNRAS 282, 1359

Humphreys, E.M.L., Yates, J.A., Gray, M.D., Field, D., Bowen, G. 2001, A\&A 379,501

Humphreys, E.M.L., Gray, M.D., Yates, J.A., et al. 2002, A\&A 286, 256

Kemball, A.J. \& Diamond, P. J. 1997, ApJ, 481, L111

Le Bertre, T. 1997, in Stellar Astrophysics (Brussels)

Menten, K.M., Melnick, G.J., Phillips, T.G., Neufeld, D.A. 1990, ApJ 363, L27

Menten, K.M., Melnick, G.J. 1991, ApJ 377, 647

Nedoluha, G.E., Watson, W.D. 1990, ApJ, 361, L53

Pardo, J. R., Cernicharo, J., González-Alfonso, E., Bujarrabal, V. 1998, A\&A, 329,219

Reid, M.J., Menten, K.M. 1990, ApJ 360, L51

Reid, M.J. \& Menten, K.M., 1997, ApJ, 476, 327

Richards, A.M.S., Yates, J.A., Cohen, R.J. 1996 MNRAS 282, 665

Richards, A.M.S., Yates, J.A., Cohen, R.J. 1998 MNRAS 299, 319

Richards, A. M. S., Cohen, R. J., Bains, I., Yates, J.A. 1999, in IAU 191, 315

Szymczak, M., Cohen, R.J. 1997, MNRAS, 288, 945

Vlemmings, W., Diamond, P. J., van Langevelde, H. J. 2001, A\&A, 375, L1

Vlemmings, W., Diamond, P. J., van Langevelde, H. J. 2002, A\&A in press

Western, L. R. \& Watson, W. D. 1983, ApJ, 275, 195

Wiebe, D. S., Watson, W. D. 1998, ApJ, 503, L71

Yates, J.A., Cohen, R.J. 1994, MNRAS 270, 958

Yates, J.A., Cohen, R.J., Hills, R.E. 1995, MNRAS 273, 529

Yi, J., Booth, R.S., Conway, J.E., et al. 2002, in prep 